Conclusion Sclerotherapy should be considered in selected patients with craniofacial (veno)lymphatic malformations as it represents a safe and successful treatment option, especially if surgical excision is considered challenging, with a high risk of complication and postoperative recurrence. Patients and parents have to be aware that sclerotherapy may require several sessions to achieve results. Generally, patients and parents are satisfied with sclerotherapy treatment outcomes, specifically the cosmetic results.

Disclosures A. Kuhn: None. A. Puri: 1; C; NIH, Stryker Neurovascular, Medtronic, Cerenovus. 2; C; Microvention, QApel, Perfuze Medical, Arsenal Medical, Merit Medical, Stryker Neurovascular, Medtronic, Cerenovus. 4; C; InNeuroCo Inc, Galaxy therapeutics, Agile Medical, Perfuze medical and NTI. C. Zoppo: None. K. de Macedo Rodrigues: None. F. Massari: None. M. Gounis: 1; C; National Institutes of Health $(\mathrm{NIH})$, the United States - Israel Binational Science Foundation, Anaconda, ApicBio, Axovant, Cerenovus, Cook Medical, Gentuity, Imperative Care, InNeuroCo, Magneto. 2; C; Cerenovus, Imperative Care, phenox, Medtronic Neurovascular, Route 92 Medical, Stryker Neurovascular. 4; C; Imperative Care, InNeuroCo and Neurogami. J. Singh: None.

\section{E-119 ANATOMICAL SNUFFBOX ACCESS (DISTAL RADIAL ARTERY) FOR CAROTID ARTERY STENTING}

${ }^{1} \mathrm{~A}$ Kuhn, ${ }^{1 J} \mathrm{~S}$ Singh, ${ }^{2} \mathrm{~S}$ Satti, ${ }^{2} \mathrm{~T}$ Eden, ${ }^{1} \mathrm{~V}$ Moholkar, ${ }^{3} \mathrm{~K}$ de Macedo Rodrigues, ${ }^{1} \mathrm{~F}$ Massari, ${ }^{1} \mathrm{~V}$ Naragum, ${ }^{1} \mathrm{~V}$ Anagnostakou, ${ }^{1} \mathrm{M}$ Gounis, ${ }^{1} \mathrm{~A}$ Puri ${ }^{*}$. 'Division of Neurointerventional Radiology, Department of Radiology and New England Center for Stroke, University of Massachusetts, Worcester, MA; ${ }^{2}$ Dept of Neurointerventional Surgery, Christiana Health System, Newark, DE; ${ }^{3}$ Greensboro Radiology, Greensboro, NC

\subsection{6/neurintsurg-2021-SNIS.214}

Purpose To report the feasibility and safety of anatomical snuffbox access (distal radial artery, dRA) for carotid artery stenting (CAS).

Methods We performed a retrospective review of our prospectively maintained neurointerventional database of CAS cases between May 2019 and February 2021. All CAS cases via the anatomical snuffbox were identified. Patient demographics, clinical information, procedural and radiographic data was collected.

Results 24 CAS procedures in 22 patients via the anatomical snuffbox were identified. Patients' mean age was 69.5 years (range 53-87 years). 4 patients were female. Mean radial artery diameter was $2.1 \mathrm{~mm}$ (range 1.6-2.8 mm). Snuffbox access was achieved in all cases. Sixteen procedures involved the right carotid artery. In 19 cases a Carotid Wallstent (Boston Scientific) was used. In 2 cases a Viabahn stent graft (Gore) was placed and a Precise stent (Cordis) was deployed in 1 case. Conversion to femoral access was required in 2 cases $(8.3 \%)$ due to persistent radial artery vasospasm resulting in patient discomfort despite multiple additional doses of intraarterial vasodilators and added intravenous sedation as well as tortuous vessel anatomy and limited support of the catheters in a type 3 aortic arch for left CAS. In our cases, dRA access was mainly obtained with a $6 \mathrm{~F}$ Prelude Ideal hydrophilic sheath introducer (Merit Medical) and procedures were mainly performed using a Benchmark catheter (Penumbra). In 3 cases a Fubuki guide catheter (Asahi Intecc) was directly inserted into the dRA over the exchange length wire (sheath-less or bareback catheter use) after removal of the

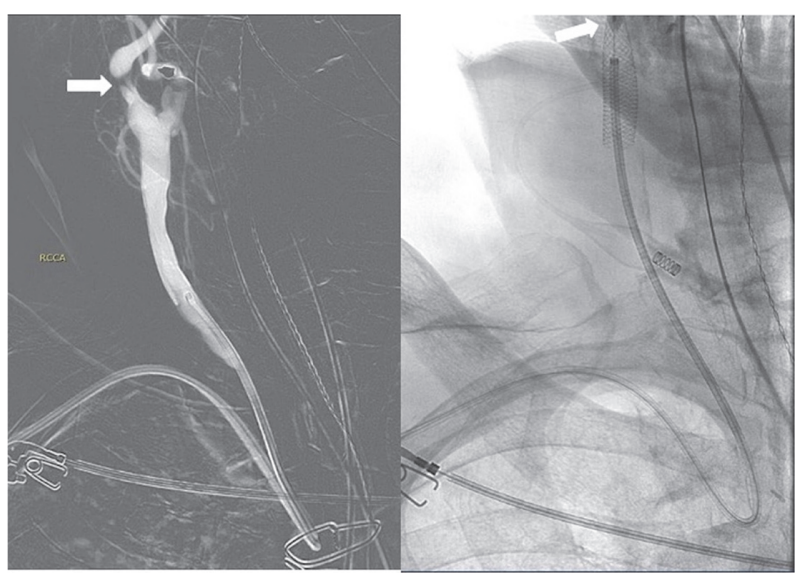

Abstract E-119 Figure 1

sheath. In one case, a $6 \mathrm{~F}$ Benchmark guide catheter was used to gain direct access to the dRA. We did not observe any peri- or postprocedural access site complications. No access site complications were noted. A cerebral protection device was used in all cases. There was no morbidity or mortality associated with any of the CAS interventions.

Conclusion Our preliminary experience with anatomical snuffbox access for CAS suggests this approach to be feasible and safe for patients. Preprocedural planning and technical considerations are both important for a successful procedure.Nevertheless, catheter systems and devices specifically designed for radial access are needed and will likely enable more interventionalists to safely perform such procedures via hand/wrist access.

Disclosures A. Kuhn: None. J. Singh: None. S. Satti: 2; C; Balt, Stryker Neuromuscular, Cerenovus, Medtronic, Microvention, Terumo. T. Eden: None. V. Moholkar: None. K. de Macedo Rodrigues: None. F. Massari: None. V. Naragum: None. V. Anagnostakou: None. M. Gounis: 1; C; National Institutes of Health (NIH), the United States - Israel Binational Science Foundation, Anaconda, ApicBio, Axovant, Cerenovus, Cook Medical, Gentuity, Imperative Care, InNeuroCo, Magneto. 2; C; Cerenovus, Imperative Care, phenox, Medtronic Neurovascular, Route 92 Medical, Stryker Neurovascular. 4; C; Imperative Care, InNeuroCo and Neurogami. A. Puri: 1; C; NIH, Stryker Neurovascular, Medtronic, Cerenovus. 2; C; Microvention, QApel, Perfuze Medical, Arsenal Medical, Merit Medical, Stryker Neurovascular, Medtronic, Cerenovus. 4; C; InNeuroCo Inc, Galaxy therapeutics, Agile Medical, Perfuze medical and NTI.

\section{E-120 FIRST PASS EFFICACY OF ANTERIOR CIRCULATION THROMBECTOMY USING THE WALRUS BALLOON GUIDE CATHETER}

${ }^{1} \mathrm{~A}$ Kuhn, ${ }^{1} \mathrm{~V}$ Naragum, ${ }^{1} \mathrm{~J}$ Singh, ${ }^{2} \mathrm{~K}$ de Macedo Rodrigues, ${ }^{1} \mathrm{~F}$ Massari, ${ }^{1} \mathrm{~V}$ Anagnostakou, ${ }^{1} \mathrm{M}$ Gounis, ${ }^{1} \mathrm{~A}$ Puri'. ${ }^{1}$ 'Division of Neurointerventional Radiology, Department of Radiology and New England Center for Stroke, University of Massachusetts, Worcester, MA; ${ }^{2}$ Greensboro Radiology, Greensboro, NC

10.1136/neurintsurg-2021-SNIS.215

Purpose Evaluation of the Walrus balloon guide catheter (BGC) first pass efficacy in mechanical thrombectomy cases compared to other guide catheters. 